\title{
Developing a ward round checklist to improve patient safety
}

\author{
Gordon Hale, Duncan McNab
}

NHS Lanarkshire, UK

\begin{abstract}
Checklists have been shown to improve care and reduce morbidity and mortality in the healthcare setting.[1] Their application in safety-critical industries outside of medicine continues to offer a strong argument for their application to medicine.[2] The daily in-patient medical ward round is a complex process and includes multiple potential risks to patient safety. This project aims to evaluate the effectiveness of a ward round review checklist on one general medical ward in a district general hospital in the UK.

A baseline audit was performed, examining case-notes for a set of pre-defined outcome measures relevant to patient safety. Compliance with documentation of each outcome measure was assessed prior to the introduction of a ward round checklist. This was followed by a quality improvement project through the use of PDSA cycles, with the aim of introducing and developing a ward round checklist over a nine month period. Following the introduction of a checklist, overall compliance with documentation of each outcome measure improved from $45 \%$ to $89 \%$.
\end{abstract}

In conclusion, a quality improvement project involving the introduction of a ward round checklist for daily use has resulted in improved documentation of outcome measures that are relevant to patient safety. Teamwork and leadership skills from clinicians committed to improving patient safety is essential to sustaining improvements in traditional ward round practice.

\section{Problem}

Ward rounds should prioritise quality, patient experience, and patient safety. However, failures in care arising from the ward round can directly affect patient safety.[3] There is wide variation in practice with regards to ward round case note documentation in our hospital. Variation in practice is more likely to lead to errors. Common issues arising from ward rounds include medication errors, poor prescription of intra-venous fluids, and omission of venous thrombo-embolism prophylaxis.

A recent review of the safety and quality of care for acute adult patients in NHS Lanarkshire, carried out by the Scottish Patient Safety Programme (SPSP), included a case note review. One of the recommendations of this report was that NHS Lanarkshire must improve communication and note keeping and improve the medical review of patients.[4]

\section{Background}

Checklists and care bundles are being used by the SPSP to address their acute adult "nine points of care priorities".[5] In the intensive care unit, daily goal setting has been adopted by the SPSP as one of their "ten essentials of safety". There have been many examples of how checklists and care bundles have been used to improve patient safety. One successful example of a care bundle already being used in NHS Lanarkshire is the "Sepsis 6" bundle which involves early identification and treatment of patients with sepsis and that has been shown to result in a reduction in mortality when adhered to.[6] Another international example of the successful use of a checklist has been the implementation of the WHO surgical safety checklist.[7] Checklists are also used in many safety critical industries outside of medicine to help standardise performance.

However, perceived barriers to checklist implementation in the healthcare setting have been identified. Examples of reasons why checklist implementation may not be easily adopted include: issues of time spent completing a checklist without perceived benefit, duplication of documentation, and poor communication among a team regarding the purpose and timing of checklist use.[8]

The ward round provides an obvious opportunity for systematically and collectively ensuring that proper standards of care are being achieved for individual patients. A joint publication in 2012 by the Royal College of Physicians and Royal College of Nursing set out gold standard recommendations for ward round practice.[9] The report acknowledges that ward rounds are complex clinical processes and that there remains significant variability in the conduct and purpose of ward rounds. The report recommends that safety checklists can reduce omissions and variations in practice while strengthening team communication, performance and patient experience. A ward round checklist can help to define a set of potential patient risks to patient safety which should be checked on a daily basis, in addition to providing a structured, replicable approach to case note documentation and clinical review.

The concept of a "ward safety checklist" has been previously described but not widely implemented.[10] While more evidence of the clinical effectiveness of a ward round checklist is needed, the evidence from the use of checklists and care bundles in other areas offers a strong argument for their use. The introduction of a structured ward round review presents a significant challenge to the existing ward round culture, and strong leadership is required to ensure that such a change to traditional practice is sustained. 


\section{Baseline measurement}

A retrospective case note review of all in-patients $(n=24)$ on a single day, after all patients had been reviewed on the ward round, on a single ward (gastroenterology) was performed. The ward round entry from that particular day was audited for compliance with the selected outcome measures. Baseline measurement data is shown in the attachment below. The average number of identified outcome measures documented in the medical notes of each patient was $45 \%$. As expected, there was a wide variation in the format and content of the case note entries.

The experience grade of the reviewer ranged from consultant to foundation year 2 doctor. While the name and grade of the reviewer were clearly documented in most cases, documentation of modified early warning score (MEWS), investigation results, diagnosis and an escalation plan in the event of deterioration were highly variable. The need for venous thrombo-embolism (VTE) prophylaxis was not documented in any ward round entry. The initial results of the baseline audit were discussed with the ward team and it was agreed that a trial of a ward round "daily goals" sheet would be performed in an attempt to improve documentation and patient safety.

See supplementary file: ds3708.docx - "Baseline audit data"

\section{Design}

The variability in ward round documentation in the case notes was felt to be due to the lack of a structured approach to documentation The suggestion for the use of a daily review checklist resulted from the successful implementation of "daily goals" in the intensive care unit and the "daily progress" sheet already being used in the medical high-dependency area in the adjacent ward. This "daily progress" sheet provided the template and was then adapted for use on the general medical ward. It was agreed among the medical and nursing team working on the ward that the trial of the "clinical review" sheet would be undertaken for use with every patient on every ward round. It was agreed that the use of the sheet would be audited at different intervals to assess compliance and to allow adaptations to be made if required. Prior to implementation of the review sheet, information was circulated to staff working on the ward to alert them to the new documentation being introduced.

\section{Strategy}

PDSA cycle 1 (November 2013): Following circulation of the proposed test of change to relevant staff working on the ward, the clinical review sheet was printed and introduced into circulation. One month following introduction, the sheet was audited using the same method as the baseline data measurement. Compliance with the sheet and documentation of the selected outcome measurements was audited. Feedback was obtained from staff engaged in the process about how the review sheet could be improved.

PDSA cycle 2 (January 2014): It was identified that some clinicians were choosing not to use the review sheet due to the lack of space available for free text. Concerns were also raised from the ward multi-disciplinary team that continuity in the notes was not always clear due to the structure of the review sheet. It was agreed that the information on the review sheet would be condensed and trialled in the form of a "sticker" as an alternative to a complete sheet. The "sticker" was trialled on one ward round on one day however following discussion with the team it was agreed that a modification to the existing sheet allowing more space for free text would be preferable and this change was made prior to re-audit.

PDSA cycle 3 (April 2014): Data collected from the first two cycles were circulated to the ward staff and the initial findings from the report were discussed at a hospital clinical governance meeting that coincided with the trust's "patient safety week". Clinician feedback prompted review of the sheet and further modification was made to allow more space for the free text clinical review while maintaining the same content in a smaller font size. Repeat data collection was performed following this further test of change. Results and findings after three PDSA cycles were presented at the hospital medical staff meeting.

\section{Results}

Following the introduction of a structured clinical review sheet, the overall compliance with the documentation of seven outcome measures improved from $45 \%$ to $89 \%$. Initial compliance with the review sheet was $70 \%$ at the point of audit one month following introduction. The use of the review sheet was sustained over a nine month period, as shown by several points of re-audit. Compliance with the review sheet improved following a series of PDSA cycles where modifications were made based on feedback from staff involved. The results and versions of the clinical review sheet from each PDSA cycle are attached.

See supplementary file: ds4671.docx - "Results"

\section{Lessons and limitations}

A structured ward round review sheet can help to standardise practice while ensuring that key components of the bedside review are not overlooked. It should be clear that the introduction of a structured ward round checklist should not be seen as a challenge to clinicians' autonomy and competence, but rather a safe-guard approach to ensure that the often perceived "basic" aspects of care are not missed. All bedside reviews should address common patient safety issues such as VTE prophylaxis, intravenous fluid prescription, drug chart review, and review of intravenous access. Prompting an individual to review such aspects can help to prevent adverse events while clear documentation of a patient's MEWS score will prompt checking of nursing observations and may enable earlier recognition of a deteriorating patient. In addition to this, a structured ward round checklist will be beneficial to the education of junior doctors reviewing patients who may be inexperienced in ward round practice, as it will prompt them to consider important aspects of care which may be easily overlooked. 
There were a number of challenges encountered with this project. Previously described barriers for the use of checklists included time required for completion, duplication of documentation, and lack of communication around purpose of the checklist.[8] Criticisms of our approach included the perception that not all aspects included in the review sheet, such as escalation plan in the event of deterioration, required review on a daily basis. It was also argued that the checklist was not applicable to every patient, such as those admitted electively for day case procedures or patients receiving end of life care in whom MEWS score documentation was no longer applicable. It was therefore accepted that it would be part of the clinician's judgement as to whether there were cases where the review sheet was not applicable and in such cases it could be omitted.

Future plans and sustainability

This trial was performed on one medical ward with a small selection of patients, although the concept of a ward round checklist is easily replicable in other areas of the hospital. Following successful introduction of the checklist in the gastroenterology ward, additional medical wards within the hospital will now be encouraged to develop their own bespoke checklist suited specifically for their needs. Early adoption of a successful patient safety initiative in the medical department of the hospital can also help to set an example and encourage use in additional departments (for example in surgical wards) with appropriate clinical governance support. Periodic audit will now be undertaken to demonstrate and review the sustainability of our intervention.

Endorsement of the checklist by senior medical and nursing staff on the ward was crucial to the success of the project. Given the frequent change-over of junior medical staff common to most NHS hospitals, the key to the long term success of this project has been through the engagement of the consultant body and the ward nursing staff who are in a position to ensure that appropriate education on checklist use occurs as doctors rotate through the ward.

\section{Conclusion}

Introducing a ward round checklist or clinical review sheet into daily practice requires strong leadership and communication skills as it represent a significant challenge to traditional ward round practice. The variation in ward round practice was evident prior to baseline audit and variation in case note documentation between clinicians reflected this. A review into patient safety at our hospital included the recommendation that communication and note keeping be improved and a structured ward round review sheet enabled clinicians to check that key patient safety issues were not being omitted from daily clinical practice. Designing and issuing a ward round checklist is unlikely to have an impact on its own as to be successful it requires a programme of communication, education, and ultimately culture change.
1. Weiser TG, Haynes AB, Lashoher A, Dziekan G, Boorman DJ, Berry WR, Gawande AA. Perspectives in quality: designing the WHO Surgical Safety Checklist. Int J Qual Health Care 2010;22(5):365-70.

2. Amin Y, Grewcock D, Andrews S, Halligan A. Why patients need leaders: introducing a ward safety checklist. J R Soc Med 2012;105:377-383.

3. Mohan N, Caldwell G. A Considerative Checklist to ensure safe daily patient review. Clin Teach 2013;10(4):209-13.

4. Healthcare Improvement Scotland. A rapid review of the safety and quality of care for acute adult patients in NHS Lanarkshire: findings and recommendations. Glasgow, 2013.

5. Scottish Patient Safety Programme. Acute Adult. http://www.scottishpatientsafetyprogramme.scot.nhs.uk/prog rammes/acute-adult (accessed $17 \mathrm{Jul} 2014$ ).

6. Daniels R, Nutbeam T, McNamara G, Galvin C. The Sepsis Six and the Severe Sepsis Resuscitation Bundle: a prospective observational cohort study. Emerg Med J 2011;28(6):507-12.

7. Haynes $A B$ et al. A surgical safety checklist to reduce morbidity and mortality in a global population. N Eng $\mathrm{J}$ Med 2009;360(5):491-9.

8. Fourcade A, Blanche JL, Grenier C, Bourgain JL, Minvielle E. Barriers to staff adoption of a surgical safety checklist. BMJ Qual Saf 2012;21(3):191-7.

9. Royal College of Physicians and Royal College of Nursing. Ward rounds in medicine: principles for best practice. London: RCP, 2012.

10. Herring R, Desai T, Caldwell G. Quality and safety at the point of care: how long should a ward round take? Clin Med 2011;11(1):20-2.

\section{Declaration of interests}

Nothing to declare.

\section{Acknowledgements}

The authors would like to thank Dr Gillian Mulholland, Dr Helen Mackie and Mr Tom Imrie for their help and support with this project.

\section{References}

